\title{
VALIDEZ JURÍDICA DE LA "PALABRA DE MATRIMONIO" EN LA VILLA Y ALCALDÍA MAYOR DE TEGUCIGALPA (1772-1822)
}

\author{
LEGAL VALIDITY OF THE MARRIAGE PROPOSAL IN THE TOWN \\ AND MAYORALTY OF TEGUCIGALPA (1772-1822)
}

José Manuel Cardona Amaya ${ }^{1}$

\section{RESUMEN}

La palabra matrimonio como una figura jurídica ocupó un lugar importante en las relaciones civiles de la época colonial hondureña, sirviendo como el primer paso formal antes de la celebración del matrimonio y, por lo tanto, con suficiente peso legal para justificar el inicio de un proceso dado su incumplimiento. En el presente artículo se explora la evolución de la validez jurídica de la palabra de matrimonio en los años finales de aplicación del derecho indiano en las colonias españolas, específicamente en la jurisdicción de la villa de Tegucigalpa y su Alcaldía Mayor. Para la investigación se utilizó el Fondo de la Alcaldía Mayor contenido en el Archivo Nacional de Honduras, encontrándose cinco casos referentes al objeto de estudio.

PALABRAS CLAVE: derecho indiano, mujeres, historia, esponsales, matrimonio

\begin{abstract}
The word of marriage as a legal figure occupied an important place in the civil relations of the Honduran colonial era, serving as the first formal step before the celebration of marriage and, therefore, with sufficient legal weight to justify the beginning of a process given its breach. This article explores the evolution of the legal validity of the word of marriage in the final years of application of Indian law in the Spanish colonies, specifically in the jurisdiction of the town of Tegucigalpa and its Mayoralty. For the research, the "Fondo de la Alcaldía Mayor" contained in the "Archivo Nacional de Honduras", finding five cases concerning the object of study.
\end{abstract}

KEYWORDS: Indian right, women, history, betrothal, marriage

Fecha de recepción: 30 de enero del 2019

Fecha de aprobación: 25 de octubre del 2019

${ }^{1}$ Docente del Departamento de Historia, Licenciado en Historia, Universidad Nacional Autónoma de Honduras,

Email: jmcardona@unah.edu.hn 


\section{INTRODUCCIÓN}

Los años finales de la dominación española en lo que hoy en día es Honduras fueron de una constante crisis y descontento entre las partes. El establecimiento del régimen de intendencias, la consolidación de vales reales y la imposición de nuevos impuestos consolidaron la impopularidad de los monarcas borbones en el territorio. Los constantes alzamientos populares, la piratería y las incursiones de poderes extranjeros se sumaron a los propios males que las políticas internas de la corona ejercían para que, finalmente, en Centroamérica, se declarase la independencia en la década de 1820.

No obstante, las reformas jurídicas de los borbones siguieron avante hasta su pérdida del control de las colonias indianas. Como indica Muro (1970, p.67), el periodo del reinado de Carlos III sería uno de perfeccionamiento de las leyes indianas, desarrollando cierta estabilidad legal y consonancia con las legislaciones castellanas. Mientras tanto, los reinados de Carlos IV y Fernando VII representan un periodo decadente (Muro, 1970, p.67) del derecho colonial, en el cual los magistrados locales tomaban cada vez más libertad en la promulgación de ordenanzas y la ignorancia de las disposiciones españolas.

En el campo del derecho eclesiástico indiano, se reuniría en 1771, después de doscientos años, el Concilio Mexicano para dictar nuevas normativas, aunque su alcance sería más limitado, al haberse separado Comayagua y Nicaragua en 1743 para unirse a la arquidiócesis de Guatemala (Pérez, Gonzáles y Aguirre, 2014, p.2).

Es en este marco de debilitación del Imperio Español que se desarrolla el presente artículo, en el cual se discute la validez jurídica de la palabra de matrimonio y lo esponsales en Villa de Tegucigalpa y, en su Alcaldía Mayor. Esta distinción geográfica es de especial importancia al considerarse la repartición de justicia en el momento histórico. Así, cuando Tegucigalpa era la cabeza de la provincia bajo el título administrativo de "Alcaldía Mayor", la autoridad suprema para materias civiles y legales le correspondía a su alcalde mayor, mientras que, durante el periodo de 1788 a 1812, cuando se suprimió esta forma y se convirtió a la Villa de Tegucigalpa en una unidad administrativa más de la Intendencia de Comayagua, le atañía al subdelegado y alcaldes ordinarios resolver estos asuntos.

En esta investigación se presenta la evolución del tratamiento judicial de la palabra de matrimonio al demostrarse como los diferentes funcionarios del Imperio Español interpretaron las leyes indianas. Utilizando documentos del Archivo Nacional de Honduras que comprenden el periodo de 1772 a 1822, se exploran cinco casos significativos sobre la materia.

\section{CONCEPTUALIZACIÓN: LA PALABRA DE MATRIMONIO EN EL DERECHO INDIANO}

El concepto de "palabra de matrimonio" en el derecho indiano puede comprenderse desde dos perspectivas, dependiendo de su ejecución: primero, en el ámbito de lo social, como una promesa informal dada verbalmente por una de las partes y aceptada por la otra y; segundo, bajo el concepto de "esponsales", en el campo de lo legal, como un contrato formal regulado por la legislación indiana y sujeto a sus normativas. Como quedará evidenciado en los casos expuestos en este artículo, en la época colonial, uno de los mayores impasses legales enfrentados por las partes era la aceptación de la palabra de matrimonio de manera verbal sin realizar las diligencias formales que exigía el contrato de esponsales.

Las fuentes del derecho para tratar asuntos de esponsales se dividen en dos: la legislación en materia eclesiástica y la legislación en materia civil. Aunque se reconocían otro tipo de uniones, el matrimonio era solamente válido in facie ecclesiae (Dougnac, 2003, p.5), por lo que los esponsales debían de realizarse según las normas establecidas por la iglesia católica. En el siglo XVIII, el Concilio de Trento culminado en 1563 continuaba siendo la norma jurídica por excelencia referida por los obispos, no obstante, de manera local, los concilios provinciales establecían las particularidades que debían aplicarse a sus sujetos. Así, a partir del Segundo Concilio Mexicano de 1554, se sometió a esta institución el obispo de Comayagua 
-que administraba sobre la gobernación de Honduras y la Alcaldía Mayor de Tegucigalpa (Pérez, Gonzáles y Aguirre, 2014, p.1).

Entre las leyes más relevantes para la materia contenidas en el III Concilio Provincial Mexicano de 1585 se encuentran las Leyes II, V y VII. La Ley II hacía ilícita la cohabitación de los conyugues previa a la bendición sacerdotal, la Ley $\mathrm{V}$ obligaba a los contrayentes a recibir su bendición nupcial solamente del sacerdote de la parroquia en la que se encontraban inscritos, mientras que la Ley VII prohibía el matrimonio a personas que no tuviesen la edad que constaba en el derecho (Concilio III Provincial Mexicano, 1859, pp.343-347), que el IV Concilio Mexicano revela ser de 14 años para los varones y 12 para las mujeres (Concilio Provincial Mexicano IV, 1898, p.177). Es de notar que estas legislaciones se aplicaban igualmente para los esponsales, no siendo válidos estos sin aprobación del párroco de las partes.

En 1776 hay una evolución en la normativa, al aprobar Carlos III la Pragmática Sanción para que los hijos e hijas de las familias pidiesen el consejo y consentimiento paterno. Esta establecía que los hijos menores de 25 años debían obtener el permiso expreso del padre o, en su falta, de la madre para poder contraer matrimonio (Dougnac, 2003, p.45). Esta ley sería incorporada al derecho indiano en 1778 mediante cédula real, siendo aplicable a todas las poblaciones de América exceptuando a los sectores más excluidos como los mulatos y los negros (Mó y Rodríguez, 2001, p.78).

En 1803, se añadió a esta norma la disposición de que ni los tribunales eclesiásticos ni civiles aceptaran demandas de esponsales sin el consentimiento paterno (Dougnac, 2003, p.46). Esta última reforma es de especial interés para el presente artículo, ya que la cédula de 1803 sería citada como respaldo legal para desestimar una demanda.

Según Dougnac (2003, pp.34-35), la validez de la palabra de los esponsales dependía de cinco requisitos: primero, que esta se haya hecho seriamente y no fingida; segundo, que esta debía ser deliberada, sin coacción ni fuerza mayor que la haya impulsado más que el deseo de contraer matrimonio; tercero, que haya sido manifestada con palabras u otro símbolo exterior y no supuesta por una de las partes; cuarto, sea aceptada por ambas partes y; quinto, que las personas estén habilitadas por la ley y cuenten al menos con siete años de edad.

Estos cinco criterios complicaban la interpretación jurídica, debido a que, si una persona expresaba palabra de matrimonio en engaño, este no estaba obligado a cumplirla, aunque recaía en un delito.

El mismo autor cita ocho motivos de disolución válida para los esponsales, entre los cuales destacan: la retractación, que se hacía por una de las partes al alcanzar la pubertad si la promesa se había hecho en la niñez; el matrimonio válido con otra persona; el pecado carnal o, copular con otra persona; el "robo" de una mujer, que involucraba estupro o violación y; por la llegada del plazo de la fecha de matrimonio sin que este se haya efectuado (Dougnac, 2003, pp.56-58).

Se debe agregar que a pesar de que una de las partes haya caído en fornicación, la otra podía solicitar continuar el matrimonio, de la misma manera, si la mujer era "robada" por otro hombre y luego devuelta, el hombre que le había procurado palabra de matrimonio podía igualmente hacerla efectiva.

\section{METODOLOGÍA}

La recopilación de fuentes para el presente artículo se realizó en el Archivo Nacional de Honduras, consultándose el Fondo de la Alcaldía Mayor de Tegucigalpa. Se revisaron 120 años de documentación, desde el año de 1700 hasta 1821, comprendiendo la limitación temporal al periodo de gobierno de los monarcas borbones en el Imperio Español, al cual pertenecía Honduras. Se encontraron cinco documentos pertenecientes a la materia, en distintos grados de preservación, desde casos completos a expedientes incompletos. El primer caso encontrado data de 1772 mientras que el último de 1822. 
El análisis de los casos se realizó utilizando las leyes de la época como referencia, sobre todo lo establecido en el III Concilio Provincial Mexicano, al cual se adscribía el obispado de Comayagua y, la aplicación de la pragmática de 1778 y el decreto real de 1803, todos estos anteriormente descritos en la conceptualización. Los casos fueron divididos en dos etapas, una primera en la que la materia de los esponsales era tratada por los juzgados eclesiásticos $\mathrm{y}$, la segunda en la cual los casos son resueltos por autoridades civiles.

\section{LA PALABRA DE MATRIMONIO COMO MATERIA ECLESIÁSTICA EN LA ALCALDÍA MAYOR DE TEGUCIGALPA (1772-1818).}

El primer caso del que se tiene constancia es una causa elevada por Agustina Gómez en contra de Cristóbal Herrera en Tatumbla, el cual porta el número de documente 1283 de la caja 214 en el fondo consultado, constando de dos folios, de las cuales tres páginas contienen el caso y una se encuentra en blanco. Agustina Gómez se presentó ante Joaquín Beltrán, cura, licenciado y juez del curato de Tatumbla, demandando se apresara a Cristóbal Herrera, quien le había prometido matrimonio (Archivo Nacional de Honduras, 1772, p.1).

Según la demandante, Cristóbal Herrera, vecino del curato del Corpus, la solicitó cuando ella estaba bajo el cuidado de su madre, vecina también de este último curato. Su hermana, Simona, le advirtió que ella había tenido "amistad ilícita"-relaciones sexuales-, previamente con ese hombre y que no era alguien con quien debía de casarse.

A pesar de esto, Agustina dejó el hogar de su madre junto a Cristóbal, partiendo para el Valle de San Lorenzo en donde convivieron un año (p.1). Allí, procedieron a prometerse esponsales ilícitamente, al realizar las diligencias el cura Manuel Antonio Cepeda, quien, al no ser el cura de sus residencias oficiales, no solamente transgredía la Ley V del Título IV del Concilio Provincial Mexicano, sino que también, no realizó la publicación de impedimentos que era requisito en la Ley IV del mismo título.
Al parecer, Cristóbal Herrera abandonó a Agustina Gómez y partió a residir a otra parte, por lo que esta solicitó la ayuda del cura de Tatumbla para que se formalizase el matrimonio prometido en los esponsales. El cura solicitó la ayuda de las autoridades, en este caso, Gerónimo de la Vega Lacayo, Alcalde Mayor y Teniente de Capitán General de Tegucigalpa, para detener a Cristóbal de Herrera (p.1). Este último dispuso que sus hombres fueran a apresar al acusado al Valle de San Lorenzo, le decomisaran los bienes y lo llevaran a Tatumbla a responder ante las autoridades eclesiásticas (p.2). El expediente del caso finaliza con el reporte de Pedro Oseguera Nolasco, quien con tres soldados fue a buscar a Cristóbal Herrera a la casa de María Antonia Velásquez, no encontrándolo allí (p.3).

El caso anterior demuestra la estrecha relación que las autoridades eclesiásticas tenían con las civiles. A pesar de que la queja debía de ser realizada ente la autoridad eclesiástica pertinente, esta debía de servirse de los magistrados civiles para asegurar a las partes, no pudiendo proceder más que en el proceso letrado. De la misma forma, evidencia el papel de los curas como funcionarios del imperio español, quienes, a pesar de ser atacados constantemente por la legislación borbónica, seguían ocupando un papel central en los asuntos civiles.

El siguiente caso es quizá un ejemplo por excelencia de como las materias legales indianas eran tratadas. El documento 2209 de la caja 240 del fondo consultado, contiene el caso de Micaela Soto contra Pedro Raudales. En 1794, Micaela Soto, vecina de Tegucigalpa, alegó que Pedro Raudales la solicitó con palabras de matrimonio y que, para su cumplimiento, le otorgó esponsales como era el procedimiento debido (Archivo Nacional de Honduras, 1794, p.1).

A pesar de esto y tras repetidas insistencias, Pedro Raudales se negaba a concretar el matrimonio, por lo que Micaela Soto, alegando que su imagen se desprestigiaba, solicitó al Alcalde Mayor hacer comparecer a su prometido y forzarle a cumplir su palabra (p.2). 
Aunque las formalidades legales parecían estar en orden, Manuel Antonio Vásquez y Rivera, regidor y alcalde ordinario de segunda nomina por Tegucigalpa, determinó que la instancia no era la correspondiente y contestó que esta petición debía de llevarse al juzgado eclesiástico para ser resuelta (p.2).

Lo anterior demuestra el respeto que los funcionarios españoles poseían en cuanto a límites de poderes legales se refiere. El Alcalde Mayor perfectamente pudo haber mandado a apresar a Pedro Raudales para iniciar el proceso, no obstante, prefirió obtener el fallo de las autoridades eclesiásticas para proceder.

El último caso para este periodo es mucho más complejo que los anteriores, ya que involucra una querella y varios testimonios cambiantes entre las partes. Se trata del caso de matrimonio de Marcelina Ayestas, contenido en el documento 2439 de la caja 245 del fondo consultado. El caso es presentado por Teresa Ayestas, hermana mayor y encargada de Marcelina Ayestas, quien tenía matrimonio tratado con José Tomás Calis. La demanda es realizada hacia dos partes, primero contra José Tomás Calis por haber disuelto los esponsales y segundo hacia Teodoro Suazo, quien presumía de haber desflorado a Marcelina y ser la causa de esta disolución (Archivo Nacional de Honduras, 1798, p.1).

La narrativa del caso se transforma a partir del testimonio de tres testigos y de la afectada. De manera resumida, lo testificado fue que, tres años antes de presentada la querella, José Tomás Calis había ofrecido palabra de matrimonio a Marcelina Ayestas, quien la aceptó (p.6).

Posteriormente, este regresó a su comandancia y, mientras tanto, Teodoro Suazo aprovechó para ofrecer esponsales a Marcelina y, bajo esta promesa, la desfloró. Cuando José Tomás Calis regresó para concretar el matrimonio, se enteró de lo sucedido y se retractó de su promesa (p.6). De allí, Teresa Ayestas, lo denunciaría por el daño hecho y lograría que lo apresaran. En un testimonio posterior, Teresa
Ayestas revelaría que el matrimonio entre su hermana y Teodoro Suazo estaba pactado para realizarse en Cantarranas, pero que este nunca llegó (p.9).

El caso culminó con Teodoro Suazo solicitando al cura y licenciado de su partido le case con Marcelina Ayestas, dando el consentimiento tanto los padres de esta como su hermana Teresa (p.11).

Los testimonios iniciales del caso fueron tomados por Don Manuel José Midence, regidor perpetuo y alcalde de primera nómina de Tegucigalpa, mientras que los finales fueron recibidos por las autoridades eclesiásticas. La materia de la querella en contra de Teodoro Suazo quedó desestimada cuando este aceptó casarse con Marcelina y dejó por escrito ante la iglesia su promesa, no solamente esto, sino que se recibió declaración de los padres de ambas partes para asegurarse de que no habría impedimentos (pp.1213).

La aceptación del matrimonio con Teodoro Suarez demuestra que la reparación del honor en un matrimonio era más importante para la sociedad colonial que los medios por los cuales se alcanzasen. Teresa Ayestas quiso compeler a José Tomás Calis a cumplir una palabra de matrimonio que no estaba en obligación legal de cumplir, debido a que su hermana había conocido carnalmente a otro hombre.

La evolución del proceso jurídico en los años finales del reino de los borbones en América queda demostrado con un caso de 1818, en el que Benito Trejo, en representación de su hija Teresa Trejo, denunció a Matías Chombo Herrera porque este la había embarazado con promesa de matrimonio. El caso es el más extenso acerca de la materia encontrado en esta investigación, constando de 33 folios, con 66 páginas en total e involucrando un representante legal letrado a favor de la parte acusada. La presentación de pruebas, la argumentación legal de las partes y la sentencia demuestran un claro viraje de lo eclesiástico a lo civil, interviniendo las autoridades de la iglesia solamente en la toma de testimonios.

Ya que Benito Trejo y su hija eran vecinos de Santa Lucía, la petición fue hecha ante el Alcalde Mayor de Tegucigalpa, quien ordenó al teniente de 
San Antonio requerir a Matías Herrera para atender el caso (Archivo Nacional de Honduras, 1818, p.1), este último fue apresado y, ya que tenía menos de 25 años, se le nombra defensor, Eusebio Ruiz (p.3) quien sería instrumental en el proceso. El primer testimonio en ser tomado sería el de Teresa de Jesús Trejo, la cual reveló haber sostenido "amistad ilícita" con Matías Herrera seis meses atrás y que, cuando lo conoció carnalmente, no se encontraba doncella pero que no había copulado con nadie más desde entonces, teniendo dos meses de embarazo (p.2).

El hecho de que Teresa de Jesús Trejo no se encontraba virgen al momento de su unión con Matías Herrera sería determinante para la sentencia. Por su lado, José Matías Herrero, ratificó lo dicho por Teresa Trejo (pp.4-5), no obstante, esclareció que él tenía todas las intenciones de casarse pero que desistió al saber que no era doncella y que era de una conducta inapropiada, al abandonar temporalmente la casa de sus padres (p.10).

El defensor legal de Matías Herrera alegó que las acciones de su representado no podían consistir estupro debido a que este no la encontró doncella al momento de copular con ella, además razonó que Trejo también tuvo relaciones sexuales con un hombre casado dispuesto a testificar en el caso (p.17). Eusebio Ruiz enviaría dos interrogatorios que servían el propósito de confirmar la mala conducta de Teresa de Jesus Trejo y que esta había sostenido relaciones sexuales con varios hombres (pp.19,29).

A partir de estos, se interrogó a los testigos, entre los cuales Dionisio Matute, Esteban Salgado, José Teodoro, Tomas Soto y Juan Matute, alegaron haber sostenido relaciones sexuales con la muchacha (pp.22-25, 31-32). En su defensa, el padre de Teresa Trejo, Benito Trejo, envió su propio interrogatorio (p.37), el cual tenía como objetivo presentar a su hija como alguien de buena conducta. Cinco personas testificaron a favor de la conducta de Teresa Trejo, no obstante, no contradijeron las alegaciones de los testigos de Matías Herrera.
Sintiendo perdido el caso, Benito Trejo se quejó ante el cabildo de Santa Lucía, alegando que los testigos de Matías Herrera conspiraban contra el honor de su hija al promulgar falsedades, con el propósito de agrandar su hombría al presumir que se habían acostado con varias mujeres (pp.45-48). La argumentación final del letrado fue que las pruebas testificales de Benito Trejo carecían de requisito de sustancia y situación y que, entre Tomas Soto y Dionisio Matute se encontraba el posible padre del hijo de Trejo, ya que ellos copularon con esta en mayo, reportándose su embarazo desde agosto (p.1).

La sentencia representa tanto la moralidad de la época, como el trato cultural hacia las mujeres. Se establece que José Matías Herrera no pudo haber violentado a Teresa Trejo porque no la tomó con fuerza ni la robó de su casa; el delito de estupro se desestimó igualmente por haberse entregado honestamente a él; los esponsales se invalidaron debido a no ser celebrados por personas en edades hábiles y, también, porque Trejo no supo guardarle fidelidad a la promesa (p.55).

La fundamentación legal de la sentencia hizo referencia tanto a una cédula de 1779 , que prohibía la condenación por estupro sin pruebas y, a la Real Cédula de 1803, en la que se establece que los esponsales debían celebrarse por personas hábiles ante las autoridades eclesiásticas pertinentes (pp.5758). El fallo definitivo absolvió a José Matías Herrera de los esponsales, no obstante, lo condenó a pagar 10 pesos a Teresa Trejo para los gastos de parto, ya que no fue posible comprobar que él no era el padre de la criatura. Posteriormente, Benito Trejo se quejaría de que este pago no se había realizado, por lo que el Alcalde Mayor de Tegucigalpa condenó a Matías Herrera a trabajar en las minas de San Lorenzo hasta pagar su deuda (pp.63-64).

Como se aprecia, el proceso de reclamo de esponsales se condujo eminentemente como un proceso civil, interviniendo los funcionarios eclesiásticos solamente en apoyo a los interrogatorios. La presencia de un letrado en defensa de José Matías 
Herrera, evidencia lo secular del procedimiento, quedando la sentencia sometido más a criterios de ley que a interpretaciones morales por los sacerdotes.

Un último caso presentado es el de la denuncia de Eugenio Lanza contra Lucas Díaz, por no cumplir su palabra de matrimonio a su hija, Manuela Cayetano. Este caso se encuentra incompleto, constando solamente de una página. La argumentación del denunciante es que Julián Díaz pensaba que José Julián Laynes se encontraba ya casado con Manuela Cayetano y que este ya la ha conocido carnalmente (Archivo Nacional de Honduras, 1822, p.1). A pesar de ser un caso presentado después de la independencia de Honduras, demuestra que la palabra de matrimonio seguía teniendo validez jurídica más allá de las leyes coloniales, hasta ser codificada propiamente en el Código Civil de 1880.

\section{CONCLUSIONES}

- Los casos presentados apoyan el argumento de que la palabra de matrimonio y los esponsales formalmente concretados eran respetados según las normas vigentes en el derecho indiano, tanto de familia como eclesiástico. Las distintas querellas y reclamos demuestran que existía un desfase entre la ley y las prácticas de la población, ya que muchas veces la palabra de matrimonio era dada y aceptada por las partes, pero invalida por no ser presentada ante un funcionario de la iglesia y consentida por los padres. De la misma manera, revelan que las mujeres fueron víctimas de personas que buscaron aprovecharse de estos vacíos legales para copular con ellas, pero también las féminas podían tomar provecho de la no concreción de los esponsales para buscar otra pareja, como lo fue en el caso de Marcelina Ayestas.

- Un dato relevante es que todas las denuncias aquí presentadas fueron interpuestas por terceras partes, lo que indica la preocupación familiar por mantener el honor y la imagen pública de sus mujeres, pero también la corta edad de las afectadas, quienes por su minoría de edad no podían denunciar de su propia cuenta.

\section{BIBLIOGRAFÍA}

- Archivo Nacional de Honduras. (1772). Documento 1283, Caja 214. Tegucigalpa: Fondo de la Alcaldía Mayor de Tegucigalpa

- Archivo Nacional de Honduras. (1794). Documento 2209, Caja 240. Tegucigalpa: Fondo de la Alcaldía Mayor de Tegucigalpa

- Archivo Nacional de Honduras. (1798). Documento 2439, Caja 245. Tegucigalpa: Fondo de la Alcaldía Mayor de Tegucigalpa

- Archivo Nacional de Honduras. (1818). Documento 2985, Caja 258. Tegucigalpa: Fondo de la Alcaldía Mayor de Tegucigalpa

- Archivo Nacional de Honduras. (1822). Documento 3389, Caja 266. Tegucigalpa: Fondo de la Alcaldía Mayor de Tegucigalpa

- Concilio III Provincial Mexicano. (1898). Concilio III Provincial Mexicano celebrado en México el año de 1585. México: Eugenio Maillafert y Compañía

- Concilio Provincial Mexicano IV. (1898). Concilio Provincial Mexicano IV celebrado en la ciudad de México el año de 1771. Queretaro: Imprenta de la Escuela de Artes.

- Dougnac, A. (2003). Esquema del Derecho de Familia Indiano. Chile: Instituto de Historia del Derecho Juan de Solórzano y Pereyra

- Mó, E. y Rodríguez, M. (2001). Pragmática Sanción de 1778: ¿solución o conflicto?. Historicas, 25(1), pp.77-108

- Muro, A. (1970). Periodificación del Derecho indiano. Revista Chilena de Historia del Derecho, (6), pp.63-67

- Pérez, L., Gonzáles, E. y Aguirre, F. (2014). Estudio Introductorio: los concilios provinciales mexicanos primero y segundo. Históricas Digital, (1), pp.1-36 